\title{
3rd Annual Conference of the Leverhulme Centre for Integrative Research on Agriculture and Health (LCIRAH), Developing methods in agriculture and health research, London, 13-14 June 2013
}

\author{
Jody Harris • Mieghan Bruce • Elisa Cavatorta • Laura Cornelsen • \\ Barbara Häsler • Rosie Green • Emily H. Morgan • \\ Sara Stevano • Helen L. Walls • Kenda Cunningham
}

Received: 31 July 2013 / Accepted: 17 September 2013 /Published online: 2 October 2013

(C) Springer Science+Business Media Dordrecht and International Society for Plant Pathology 2013

\section{Introduction}

Agriculture and health interact in complex ways via food systems and nutrition, creating positive and negative synergies, which differ according to economic, political and environmental contexts, but particularly affecting the lives of vulnerable populations in low-income countries. Research in these subjects has historically proceeded in relative isolation, with different disciplines applying distinct methods to

J. Harris $\cdot$ M. Bruce $\cdot$ E. Cavatorta $\cdot$ L. Cornelsen $\cdot$ B. Häsler $\cdot$ R. Green · E. H. Morgan · S. Stevano $\cdot$ H. L. Walls $\cdot$ K. Cunningham Leverhulme Centre for Integrative Research on Agriculture and Health (LCIRAH), London, UK

\section{J. Harris}

International Food Policy Research institute (IFPRI),

Washington DC, USA

L. Cornelsen · R. Green • E. H. Morgan · H. L. Walls •

K. Cunningham

London School of Hygiene and Tropical Medicine (LSHTM), London, UK

J. Harris $\cdot$ E. Cavatorta $\cdot$ S. Stevano

School of Oriental and African Studies (SOAS), London, UK

H. L. Walls

Australian National University (ANU), Canberra, Australia

M. Bruce $\cdot$ B. Häsler

Royal Veterinary College (RVC), London, UK

J. Harris $(\square)$

LCIRAH, London International Development Centre,

36 Gordon Square, London WC1H 0PD, UK

e-mail: jody_harris@soas.ac.uk generate knowledge and inform sector-based policy and practice. This segregated working is not beneficial if research is to inform the design and implementation of programs and policies that aim to fully and sustainably address the nexus of agriculture and health. Encouragingly, policy and programmatic initiatives, as well as research endeavours, have been increasingly attentive to the linkage of agriculture and health as a method for improving nutritional status and health (Masset et al. 2012). More and better multi-sectoral action will be necessary, however, to reduce the harms and enhance the benefits of the agriculture-health relationship, and research increasingly needs to engage with this complex picture if findings are to remain relevant to policy and practice.

In response to this interdisciplinary challenge, the Leverhulme Centre for Integrative Research on Agriculture and Health (LCIRAH) was established in 2010 with a grant from the Leverhulme Trust to develop "unifying approaches and methodologies for understanding the relationship between agricultural production and population health, and the factors which drive them both"- an agri-health research agenda (www.lcirah.ac.uk). Since its inception, LCIRAH has hosted three international conferences endeavouring to bring together experts from different sectors to share perspectives and explore integrated inter-disciplinary approaches to global agri-health challenges.

In 2011, the first conference, organised with the International Food Policy Research Institute (IFPRI), had a strong focus on agri-health metrics, with scholars presenting work on ways in which metrics and methods can cross disciplines to fill knowledge gaps. The conference found a potentially huge benefit in promoting the understanding and utilization of different methods across disciplines to move the agri-health research agenda forward. The second conference, in 2012, 
focused on the role of agriculture, food systems, nutrition and health in the demographic and dietary transitions in low- and middle-income countries, and the emerging issue of noncommunicable disease (NCD) in these populations. The conference expressed a strong sense of urgency, given that many elements driving the rapid rise in NCDs can be addressed now through effective advocacy and action. It also concluded that further research is critical to establishing the evidence for changes in food systems and policy and for guiding locally relevant interventions. Reports from both of these conferences are available on the LCIRAH website.

This is a report of the third annual LCIRAH conference, held in London in June 2013 with a focus on innovative methodological and integrative aspects of agri-health research. The conference featured the work of researchers in agricultural development, nutrition, public health, economics, anthropology, veterinary science and other related fields. Researchers were asked to present innovative methods in either on-going or completed research in agri-health; not all presentations reported below therefore presented results. This conference also created the opportunity for sharing agri-health research initiatives via poster presentations, which covered a range of methods for assessing topics from home gardening in Cambodia to the availability of nutrients from different supermarkets in Colombia. This year, the conference particularly welcomed early career researchers or researchers new to the field. However, as noted by the conference host and LCIRAH director Jeff Waage in his welcome address, in such a new discipline as agri-health, even the most seasoned academic can be a novice!

\section{Day 1}

Putting agri-health research in the driving seat

Jeff Waage (LCIRAH) referenced two key events in his welcome address - the 'Nutrition for Growth' summit held in the run-up to the G8 talks, and the launch of the 2013 Lancet series on maternal and child under nutrition - both held in London the previous week. The Nutrition for Growth summit led to the creation of a global panel on agriculture and food systems for nutrition, a UK government initiative to be chaired by Sir John Beddington, former UK government chief scientific advisor, and John Kufour, former President of Ghana. The London International Development Centre (LIDC), which hosts LCIRAH and is directed by Waage, will serve as secretariat to this high-level panel, which aims to synthesize evidence on the role of agriculture in nutrition to feed into efforts to reduce under-nutrition globally over 3 years. The Lancet series found agriculture and food systems to be key to eliminating undernutrition (Pinstrup-Andersen 2013; Ruel and Alderman 2013), though Waage noted challenges for the academic community in improving understanding of these complex issues among fellow researchers and policymakers, given the limited evidence base and frequency of work in sectoral isolation. Taken together with a recent groundswell of interest and action around under-nutrition and the links between agriculture and health, these events provide a positive backdrop for the theme of the conference and the work of LCIRAH.

Alan Dangour (LSHTM, LCIRAH) followed the introduction with a keynote address. Dangour noted that much agri-health research to date has been 'agri-health by accident', with researchers from different disciplines studying important questions relevant to this nexus, but rarely identifying this work as a single body of evidence. He argued that due to a growing literature, and the pressing nutrition and health issues linked to agriculture, there is now a need to put agri-health in the driving seat. Bringing together recent evidence and tales from his own experiences in agri-health research, he highlighted the importance of carefully conceived questions, well-designed studies, and clear communication of findings to bring the field together into a coherent body of work. To achieve this, Dangour highlighted the need for innovation in problem solving and encouraged conference participants to capitalize on international momentum to address agriculture and health challenges for development.

Session 1: empirical methods: analysing nutrition and food consumption

Emmanuel Skoufias (World Bank) chaired this session focusing on novel empirical analysis of nutrition and food consumption using existing large datasets. Most of the work showed how existing data could be collated and used to answer important agri-health research questions.

Anna Herforth (Independent consultant) opened the session by presenting results of a UN Food and Agricultural Organisation (FAO) survey of researchers currently conducting agriculturenutrition studies and evaluations, building on results from a 2012 mapping study done by LCIRAH. The aim was to understand how agriculture projects will measure impact on nutrition, so that discussions of the evidence base can be informed about the current generation of new evidence. She observed improved attention to impact pathways and measurement of dietary outcomes, while noting that the scope of these studies is mostly limited to measurement of impact on producer households, not on the wider food system; and mostly in relatively small projects where improving nutrition is the main objective. A different approach is likely to be needed to assess larger agriculture programs or investments where nutrition is not necessarily the primary goal. Herforth proposed an approach for monitoring and evaluation (M\&E) of nutrition impact in such larger programs, where capacity and incentives for high-quality $\mathrm{M} \& \mathrm{E}$, particularly for nutrition, may be limited without 
external support. The method proposed involves the regular collection of quantitative and qualitative data in geographically representative sentinel sites and comparable sites in non-project areas. Data collected would include information regarding: 1) the extent to which households have been affected by the intervention; 2) household food security; 3) dietary quality and, if appropriate, nutritional status of young children or other household members; 4) other factors which may indicate harm to food security or nutrition; and 5) indicators of primary interest to project managers. In addition to its value for understanding program impact, information collected is envisioned to feed back into program implementation to minimize harms and maximize benefits, both for nutrition and other goals (e.g. income, sustainability).

Laura Cornelsen (LSHTM, LCIRAH) presented the results from a systematic review and meta-analysis of global foodprice elasticities, essential to improving understanding as to what extent changes in food prices affect food consumption. This research appears to be the first attempt to estimate ownprice and cross-price elasticities globally. Using data extracted from a systematic review of the existing literature, the paper used meta-regression to consistently estimate the average response of price changes in various food groups. The results pointed to an inelastic demand for foods in general, but a response in demand to changes in food prices was most pronounced in low-income countries for foods such as meat, dairy and fish. There were also important patterns of substitution (cross-price elasticities) that can undermine or reinforce the impact of a change in a particular food price.

Elisa Cavatorta (SOAS, LCIRAH) presented a study on child under-nutrition in India, a country with wide geographical variation in child stunting across states. She asked what the driving forces behind disparities in child stunting across Indian states are, and whether disparities are due to differences in underlying determinants of nutritional outcomes - such as household income, maternal education or agricultural variables - or due to differences in the strength of association between those determinants and nutrition in each location. Cavatorta described an empirical methodology based on counterfactual decomposition, which simulates the conditions in successful versus unsuccessful states, in order to assess what proportion of malnutrition is due to household determinants versus structural determinants. The results pointed to the importance of wealth and maternal education as key drivers of nutritional gaps across Indian states and, to some extent, agricultural variables such as livestock ownership as additional determinants. The method is currently being further refined.

Andrew Jamali (National Statistical Office, Malawi) presented an on-going study on the effect of climate change on child nutrition in Malawi, this being a country of predominantly rainfed agriculture and hence sensitive to climatic conditions.
Using a novel combination of various available datasets spanning 15 years, the author discussed an empirical approach to model the determinants of nutritional outcomes and elaborated on the impact of various climatic scenarios using multi-level modelling. The study hopes to highlight the consequences of unusual temperature and rainfall patterns on agriculture and then on nutrition in Malawi.

Seifu Hagos Gebreyesus (Addis Ababa University, University of Bergen) discussed the effect of changes in rainfall patterns on crop availability and child malnutrition in Ethiopia. The authors constructed an original zone-level dataset including data on crop production, livestock production, child nutrition and rainfall. Using panel data techniques, preliminary results indicated little direct association between rainfall and malnutrition, identifying issues with ecological fallacy, exposure assessment, complexity and nonlinearity.

In conclusion, the session demonstrated a wide range of data and methods applicable to the study of agriculture, food systems and nutrition. However, the paucity of integrated datasets, incomplete modelling of the known determinants of child under-nutrition, and credibility of some data remain important issues. There is scope and need for generating more empirical evidence about which of the various conceptual pathways between agriculture and nutrition are leveraged by agricultural and agri-sensitive projects, and strong conceptual frameworks should inform the design of future empirical studies.

Session 2: Randomized control trials and other methods for assessing interventions

Patrick Webb (Tufts University) chaired this session. Although considerable scope exists for agricultural interventions to positively impact nutritional status and health, agricultural interventions cannot yet be assumed to improve the nutritional status of populations. A recent systematic review could not reach a conclusion on the effectiveness of agricultural interventions on child nutritional status due to methodological weaknesses, such as lack of randomisation and adequate control groups, as well as insufficient statistical power and outcome measures of the studies (Masset et al. 2012). Similarly, a systematic review by Dangour et al. found a paucity of robust evidence of the impact of agricultural policies on nutrition and health through changes in food prices (Dangour et al. 2013). The presentations in this session addressed some of these issues by looking at the health and nutritional impacts of biofortification, household food production and security, smallscale aquaculture in homestead food production and school feeding programmes in various Asian and African countries.

Alan de Brauw (IFPRI) presented findings from an evaluation of a project that piloted dissemination of a bio-fortified crop, 
provitamin-A-rich orange-fleshed sweet potato (OFSP), as a strategy to reduce vitamin A deficiency. The project evaluated two models of intervention in Mozambique and Uganda with different costs and intensity using a cluster-randomized control trial of vine delivery and education sessions. Causal mediation analysis was used in the absence of randomization of consumption to examine impact pathways. The two intervention types assessed yielded similar results and in both countries the project led to high adoption rates and nearly doubled average dietary intake of provitamin A. Mothers' knowledge increased, as did growing of OFSP vines, but the narrow measures of education messages designed to increase demand did not affect OFSP adoption or consumption; consequently, project success was largely attributable to the promotion of crop adoption rather than behavioural change.

Adewale Oparinde (IFPRI) presented an assessment of consumer acceptance of bio-fortified staple crops in India and Nigeria, which was investigated using sensory evaluation and an experimental auction technique, where real money was exchanged for real goods in simulated markets. The investigators examined consumer preferences including taste, aroma and colour and willingness to pay for bio-fortified varieties compared with conventional ones, given different levels and sources of information. They also examined how nutritional information length and content, brands and logos, certifying agency and trustworthiness of the agency delivering the planting material affected consumer acceptance. Results related to sensory attributes, certifying agency and nutrition information varied by country, but provided context-specific information on market delivery of novel foods.

Swetha Manohar (Johns Hopkins University) presented the design and methods of a planned national assessment of agrihealth pathways in Nepal. The study plans to collect household level data on maternal and child anthropometrics, diet, health and participation in health, nutrition and microcredit services, as well as socioeconomic status, programme participation, and agricultural production through three annual panel surveys of preschool aged children and their mothers. Community-level data using geographical information systems will map and characterise features such as markets and food prices, health and agricultural services and infrastructure. The study will provide estimated and modifiable linkages between maternal and child diet and nutritional status and indices of food security and programme participation. Repeat, annual surveys are expected to reveal directions and extents of changes, with seasonal data emerging from sentinel sites, to inform and guide type, content and coverage of programmes seeking to improve food security and nutrition in Nepal.

Tim Green (University of British Columbia) presented the methodological plan of an on-going assessment of plant-based homestead food production (HFP). This 22-month cluster randomized control study evaluated the effect of plant-based HFP, with and without aquaculture, on nutritional outcomes in Cambodia. Clusters were randomly assigned to one of three groups: plant-based HFP, HFP plus aquaculture, or control. The study consisted of baseline and endpoint surveys including indicators of anthropometry and biochemical markers, household food security, dietary diversity, food production, and income generation, and allowed for different timescales to see impact on these.

Elly Weke and Craig Cohen (University of California San Francisco and Kenya Medical Research Institute) presented findings from a pilot study of a multi-sectoral sustainable agricultural intervention designed to improve health, including HIV-related health. Participants, with access to surface water and land, were selected from two clinic-based facilities and sites were randomly assigned to intervention and control. Data were collected on household economic and agricultural indicators, food insecurity, physical and mental health status, healthcare utilisation and drug adherence, HIV-related stigma, HIV risk behaviours, anthropometry and empowerment. A conceptual model for the intervention informed this pilot study that evaluated intermediate factors on the causal pathways; a final randomised control trial will look at HIV outcomes and transmission behaviours. Process evaluation data were also collected to assess the feasibility and acceptability of the study components. Initial results of the impact of the intervention on household food security, income and mental health will be released in the spring of 2014 .

Aulo Gelli (Imperial College) presented the design of a randomised control trial examining the impact of school feeding on nutrition, education, agriculture and other social outcomes in Ghana using a mixed method approach, including child, household, school and community level surveys as well as focus group discussions with project stakeholders. The evaluation will examine the impact of linking the provision of school food service to smallholder farmer income, as well as the link between community level engagement and programme performance. The randomly assigned interventions include government-implemented school feeding programmes and home-grown school feeding and social accountability groups, including schools and communities where the standard programme will be implemented alongside an innovative pilot project aimed at enhancing nutrition, agriculture and social accountability. The study will look at outcomes such as smallholder farmer income, child health and nutritional status and school participation and learning. Other outcomes will include social accountability and small to medium enterprise development in provision of school food services.

In conclusion, this session demonstrated the use of randomised controlled designs and other methods in evaluating the impact of agricultural interventions on nutritional status and health. Despite the considerable challenges in the evaluation of such interventions, evaluation is crucial to measuring the impact of 
interventions, and further development of this area will be important for informing best practice (Dangour et al. 2013).

\section{Day 2}

John McDermott (IFPRI), Director of the CGIAR Research Program on Agriculture for Nutrition and Health (A4NH), opened day two of the conference with a keynote address in which he described the A4NH research portfolio and identified emerging opportunities and challenges in the agri-health field. Research areas within A4NH include bio-fortification, agriculture-associated diseases, value chains for enhanced nutrition and integrated agriculture, nutrition and health programs and policies. McDermott said that since the commencement of A4NH in 2011, nutrition and health have gained a higher profile in agricultural development efforts and throughout the CGIAR (Consultative Group on International Agricultural Research) system. Continued capacity development is necessary to scale-up and sustain agri-health activities, which are where $\mathrm{A} 4 \mathrm{NH}$ places a particular emphasis. As the program matures, changes are being made to strengthen partnerships including making an increased share of the budget available to partners. McDermott urged conference participants to continue working to forge new partnerships and work with partners to strengthen capacity and coordination.

Session 3: new methods: food consumption and nutrition

Milla McLachlan (Stellenbosch University) chaired this session focusing on innovative methods to study and assess food consumption and nutrition broadly, as well as links to agriculture. Methods normally used to assess diet and nutritional status range from surveys of food consumption to anthropometric measures of children. Whether considered generally or specifically through their linkages with agriculture, diet and nutrition prove difficult to measure because of their multidimensional nature. The papers presented in this session addressed the need to both expand and refine methods of collecting data on food, nutrition and agriculture-nutrition linkages.

Melissa Antal (The Manoff Group) opened the session by presenting a new multidimensional index called HAND (Household Agriculture and Nutrition Development Index). While nutritional education programmes tend to be characterised by a high level of generality, HAND brings rigour to behaviour change communication by focusing on five key behaviours for household access to high quality foods. The HAND framework targets aspects of food acquisition for a programming context and includes a counselling tool at the household level, and a scoring index serves to provide a goal for change for households or their communities. The tool can be adapted according to context and field tests are currently being conducted.

Julien Chalimbaud (Action Contre la Faim) described the Nutrition Causal Analysis method, under development by $\mathrm{ACF}$, which has been developed to address the weaknesses of many analyses of the complex and multi-factorial determinants of under-nutrition. While common conceptual frameworks of malnutrition causality are clear, these frameworks are not well adapted for field research. The Nutrition Causal Analysis (NCA) method is a holistic, cost-effective analytical approach whose strength lies in synthesizing, triangulating, and rating many disparate sources of data in order to generate stakeholder dialogue, and hopefully consensus, around the causes of malnutrition in a particular locality. A 4-month pilot study in Burkina Faso revealed the ways in which seasonality influences disease and under nutrition patterns, and that women's farming activities may have negative impacts on child care practices.

Fabrice DeClerck (Bioversity International) with Byan Luckett and Diego Rose of Tulane University, and Jessica Fanzo of Columbia University put forward an innovation in showing how the ecological tool of functional diversity can be applied to nutrition. This method originated from early work on "eco-nutrition" which sought to understand the complex relationship between agriculture, environment and human health. Functional groups are formed by foods that have the same function (i.e. contain the same nutrients of interest); the degree of functional nutrition diversity in an agro-ecological zone is determined by the presence of foods containing different nutrients, rather than many foods containing the same nutrient. Generally, increasing species richness increases nutritional functional diversity, but previous research has found that this is not always true among farmer's fields in Africa. The method presented permits researchers to explore social (gender, age, or livelihood) and spatial (rural vs. urban, distance to markets, agro-ecological zones) characteristics and how they influence the impact of dietary diversity on human nutrition. DeClerck and colleagues are currently exploring these relationships by applying the functional diversity measure to the 2010-2011 Malawi Living Standards Measurement Study. This approach can help to identify the pathways and conditions through which dietary diversity has the greatest impact, and will provide insights on how best to target agricultural interventions, help direct extension activities to improve diets, and inform policy formulation.

Lizzie Hull (SOAS, LCIRAH) provided an introduction to the role of anthropology and ethnographic research methods in agri-health research. Hull noted that participant observation, as one example of these methods, is ultimately required in order to answer several types of research questions for which 
quantitative methods are not appropriate. Qualitative research such as this makes use of deductive reasoning from empirical observation to theoretical abstraction; in this sense, anthropological research provides information on categories and definitions that are otherwise assumed, particularly in survey work, and can inform further quantitative work or stand alone. This point was illustrated by an example taken from research conducted in KwaZulu-Natal, South Africa, where agricultural production has gone through an individualisation process associated with changing capacity to mobilise family labour and decreasing marriage rates. These processes of change suggest that the household may not be the most appropriate unit of analysis, as households appear to expand and contract in relation to food acquisition practices and food security status. Anthropology in agri-health research can therefore systematically describe complexity at a local level, which is often missed in other work.

In summary, this session demonstrated that the multidimensional character of food consumption and nutrition practices requires innovative methods and approaches in order to capture the complexity of the interactions. All of the papers highlighted the importance of addressing determinants, pathways and processes in methods used to assess diets, nutrition and their links with agricultural activities and interventions.

\section{Session 4: new methods: food systems}

Anthony Costello (University College London) chaired this session, which considered new approaches for examining food systems. If poor health and nutrition are to be mitigated, uncovering and understanding fundamental determinants of these phenomena is a pre-requisite. By looking across a system and studying not only its individual parts but also their interactions, identification of the most effective leverage points becomes possible (Meadows 1999). The papers presented focused on emerging agri-health methods that transcend traditional disciplinary silos and take a systems approach.

Edi Basuno (Ministry of Agriculture, Indonesia) opened the session by describing the importance of adopting an eco-health perspective in agri-health research, which considers how biological, physical, social and economic systems interact. To illustrate the application of an eco-health perspective, he presented the methods used in a multi-country study exploring the impact of poultry production clusters on smallholder livelihoods in South-East Asia. Basuno described how policy responses to highly pathogenic avian influenza outbreaks have led to significant changes in the structure of poultry production in the region, with a move away from small-scale production. The research employed participatory community-based action methods to collect both qualitative and quantitative data that can be used to formulate policy recommendations to promote sustainable livelihoods for smallholder producers.

Roseline Remans (Columbia University) introduced the Vital Signs Africa integrated monitoring system on agricultural landscapes, co-led by Conservation International, the Earth Institute at Columbia University and the Council for Scientific and Industrial Research in South Africa. The aim of Vital Signs is to provide integrated information on agriculture, ecosystems and human wellbeing that can be used to inform agricultural development decision-making and monitor outcomes in a more holistic way. The open-access system aggregates data at multiple levels (from the household to regional scales) into decision-support indicators (e.g. local food sufficiency index, adequate water supply), which will be presented through a customizable tool for policymakers. Implementation of the system is currently underway for large regions in Tanzania, Ghana, and Ethiopia. A principal goal of this first stage is to build a system and the necessary capacity for integrative data collection, analysis and use. It is hoped that the experience gained in these three countries will lead to broad adoption of the system in Africa and elsewhere.

Barbara Häsler (Royal Veterinary College, LCIRAH) discussed the importance of integrating food safety and nutritional assessments in livestock and fish value chains. While the growth of the livestock and fish sector in low- and middleincome countries provides opportunities for poverty alleviation and improvements in nutrition and health, it also brings increased risk of foodborne disease. To be able to advise value chain stakeholders and participants about pro-poor interventions in livestock and fish value chains, the International Livestock Research Institute (ILRI) required a tool for the rapid integrated assessment of food safety and nutrition. To develop the tool, mixed methods research informed the design of a conceptual framework outlining the links between food safety, nutrition and economic value in livestock and fish value chains. The framework calls for the collection of a combination of economic, epidemiological, social and nutrition data and was applied to three value chain case studies: tilapia in Egypt, dairy in Tanzania, and pork in Vietnam. Preliminary results from Tanzania showed high consumer interest in increased milk consumption and identified constraints to production at farm level, such as limited technical knowledge and the availability of dairy breeds. Further, findings showed limited incentives for improved food safety practices in milk production and sale, thus highlighting the importance of considering trade-offs between food safety and nutrition in livestock value chains.

Georgia Ladbury (University of Glasgow) presented methods for examining zoonotic disease risks along dairy value chains in Tanzania, particularly those chains supplying dairy to urban 
residents. The research involves a value chain risk analysis focused on uncovering where and by whom the different value chain activities are being carried out, what the major disease risks are, why they arise, and how they can be reduced. The study mixes qualitative data collection with epidemiological and lab analyses. She dedicated the second part of her presentation to a discussion of the practical challenges of conducting inter-disciplinary research - such as that required in agri-health - for early career researchers, such as finding suitable supervisors and work locations, and difficulties of communication across disciplines.

Overall, the presentations and subsequent discussion highlighted the importance of building inter-disciplinarity into research design and demonstrated the value of mixed methods approaches for studying food systems. The use of various qualitative and quantitative methods in tandem can help in addressing different aspects of a research question or add depth to findings (Green and Thorogood 2004). In so doing, they may uncover solutions to complex agri-health challenges and support the design of integrated interventions and policies.

\section{Conclusions}

This conference aimed to bring early-career researchers from many different fields together to discuss agri-health research methods; conference presentations reflected the broad range of methods and metrics currently in use. Conclusions can be drawn on themes in research methods emerging from the conference, and more broadly on lessons learned for interdisciplinary conferences in the future.

Almost every presentation introduced a conceptual framework in order to place its analysis in the broader context of complex agri-health dynamics. Many steps along the causal pathways from agriculture to health were covered across the range of projects presented, but the focus of most measurement was on agricultural variables or nutritional status, rather than the wider food system or health. Several studies used modelling to tease out the contribution of different factors to nutrition, health or food safety; however, greater conceptual sophistication (for instance addressing each stage in a known impact pathway, or each known determinant of an outcome) is needed when designing these models, so as not to miss potentially important confounding factors.

There remains a lack of integrated datasets containing both agriculture and health information, although this disconnect has been recognised before (Gillespie et al. 2012). Alongside this is the need for clarity on definitions of concepts used in research, and robust study design elements such as consideration of adequate sample sizes and clear and logical methods. Conference debates surfaced regarding the importance of engaging program designers during research design where evaluations are being undertaken to foster understanding. Finally, some fields, even outside agriculture- or healthrelated disciplines, are further advanced in their methods for assessing certain issues, and the agri-health community would do well to bring in learning from these disciplines.

Although this type of inter-disciplinary conference exemplifies the challenges of communicating ideas and methods across disciplines, it also stimulates interesting discussions and learning about other fields. Researchers along the full spectrum of experience and from all world regions exchanged ideas for future agri-health research and expressed their anticipation for future workshops, seminars, and other networking and learning opportunities. Participants expressed interest in following up on the findings from the studies presented, particularly via networking opportunities to be provided through a proposed 'Agri-Health Academy' series of workshops and seminars to be led by LCIRAH and A4NH. The conference urged a continued dialogue on how agri-health research can be translated into programme and policy recommendations.

In the closing session, the conference was summarized by Suneetha Kadiyala (LCIRAH). The multiple methods and frameworks presented at the conference are clearly a starting point on a quest for clarity in a highly complex field. The conference identified a need for new tools, and better and consistent application of existing tools and frameworks across disciplines; higher quality and better availability of integrated data across subjects; sounder research conceptualization; and greater understanding of mechanisms and incentives for multidisciplinary research and multi-sectoral work. The current interest in the role of agriculture and food systems in nutrition and health provide a strong platform for improving and expanding agri-health research in the medium term, with hopes for positive impacts on population health in the longer term.

Acknowledgments This work has been partly supported by the Leverhulme Centre for Integrative Research on Agriculture and Health (LCIRAH). All authors declare no conflicts of interest. JH, MB, EHM, SS, HLW contributed to writing sections of the manuscript, JH and $\mathrm{KC}$ prepared the final manuscript and LC, BH and RG reviewed the manuscript and provided critical edits.

\section{References}

Dangour, A. D., Hawkesworth, S., Shankar, B., Watson, L., Srinivasan, C. S., Morgan, E. H.et al. (2013). Can nutrition be promoted through agriculture-led food price policies? A systematic review. British Medical Journal, 3(6). doi:10.1136/bmjopen2013-002937

Gillespie, S., Harris, J., \& Kadiyala, S. (2012). The Agriculture-Nutrition Disconnect in India What Do We Know? Washington D.C.

Green, J., \& Thorogood, N. (2004). Developing qualitative research designs. In Qualitative Methods for Health Research. London: Sage. 
Masset, E., Haddad, L., Cornelius, A., \& Isaza-Castro, J. (2012). Effectiveness of agricultural interventions that aim to improve nutritional status of children: systematic review. British Medical Journal, 344, d8222. doi:10.1136/bmj.d8222.

Meadows, D. (1999). Leverage Points: Places to Intervene in a System (pp. 1-21). Hartland, VT.
Pinstrup-Andersen, P. (2013). Nutrition-sensitive food systems: from rhetoric to action. The Lancet, 6736(13), 6-7. doi:10.1016/S0140-6736(13)61053-3.

Ruel, M. T., \& Alderman, H. (2013). Nutrition-sensitive interventions and programmes: how can they help to accelerate progress in improving maternal and child nutrition? The Lancet, 6736(13), 116. doi:10.1016/S0140-6736(13)60843-0. 